\title{
UNIVERSITYOF
}

FORWARD

THINKING

WESTMINSTER用

WestminsterResearch

http://www.westminster.ac.uk/westminsterresearch

Deferoxamine and Curcumin Loaded Nanocarriers Protect

Against Rotenone-Induced Neurotoxicity

Mursaleen, L., Somavarapu, S. and Zariwala, M.

This is a copy of the accepted author manuscript of a chapter published in the Journal of Parkinson's Disease, 10 (1), pp. 99-111. doi:10.3233/JPD-191754.

The final publication is available at IOS Press through:

https://dx.doi.org/10.3233/JPD-191754

The WestminsterResearch online digital archive at the University of Westminster aims to make the research output of the University available to a wider audience. Copyright and Moral Rights remain with the authors and/or copyright owners. 


\section{DEFEROXAMINE AND CURCUMIN LOADED NANOCARRIERS PROTECT AGAINST ROTENONE INDUCED NEUROTOXICITY}

Leah Mursaleen 1, 2, 3, Satyanarayana Somavarapu 2, and Mohammed Gulrez Zariwala ${ }^{1^{*}}$

${ }^{1}$ School of Life Sciences, University of Westminster, 115 New Cavendish Street, London, W1W 6UW, UK.

2 Department of Pharmaceutics, UCL School of Pharmacy, 29-39 Brunswick Square, London, WC1N 1AX, UK.

${ }^{3}$ The Cure Parkinson's Trust, 120 Baker Street, London W1U6TU, UK.

*Corresponding author:

Dr M. Gulrez Zariwala

School of Life Sciences, University of Westminster

115 New Cavendish Street, London W1W 6UW

zariwam@wmin.ac.uk

0044 (0)20 79115000 Internal Ext: 65086

Key words: Parkinson's disease, Nanotechnology, Curcumin, Deferoxamine, Oxidative Stress 


\section{ABSTRACT}

Introduction: Reduced glutathione and excess free iron within dopaminergic, substantia nigra neurons in Parkinson's disease (PD) can drive accumulation of toxic hydroxyl radicals resulting in sustained oxidative stress and cellular damage. Factors such as brain penetrance and bioavailability have limited the advancement of potential antioxidant and iron chelator therapies for PD.

Objective: This study aimed to develop novel nanocarrier delivery systems for the antioxidant curcumin and/or iron chelator deferoxamine (DFO) to protect against rotenoneinduced changes in cell viability and oxidative stress in SH-SY5Y cells.

Method: Nanocarriers of curcumin and/or DFO were prepared using Pluronic F68 (P68) with or without dequilinium (DQA) by modified thin-film hydration. Cell viability was assessed using an MTT assay and oxidative stress was measured using Thiobarbituric acid reactive substances (TBARS) and cellular antioxidant activity (CAA) assays.

Results: All formulations demonstrated high encapsulation efficiency (65-96\%) and nanocarrier size was $<200 \mathrm{~nm}$. 3h-pretreatment with P68 or P68+DQA nanocarriers containing various concentrations of curcumin and/or DFO significantly protected against rotenone-reduced cell viability. The addition of DFO to curcumin-loaded P68+DQA nanocarriers resulted in increased protection by at least $10 \%$. All nanoformulations significantly protected against rotenone-induced lipid peroxidation $(p<0.0001)$. The addition of DQA, which targets mitochondria, resulted in up to $65 \%$ increase in cellular antioxidant activity. In nearly all preparations, the combination of $10 \mathrm{uM}$ curcumin and 100uM DFO had the most antioxidant activity. 
Conclusion: This study demonstrates for the first time the formulation and delivery using P68 and P68+DQA curcumin and/or DFO nanocarriers to protect against oxidative stress induced by a rotenone PD model. This strategy to combine antioxidants with iron chelators may provide a novel approach to fully utilise their therapeutic benefit for PD.

\section{Introduction}

Post mortem analysis and MRI techniques have highlighted a marked accumulation of free iron within the substantia nigra (SN) in Parkinson's disease (PD) [1-6]. This, together with reduced levels of the natural antioxidant glutathione, can drive accumulation of toxic hydroxyl radicals within mitochondria resulting in sustained oxidative stress and catastrophic cellular damage $[7,8]$. Iron-induced oxidative stress is likely a key contributor to the neurodegeneration observed in PD since intra-nigral injection of iron in rats induces both a PD-like phenotype and pathology [9]. Elevated iron deposition in the SN of people with PD have been significantly correlated with motor symptoms and some non-motor symptoms such as cognitive, sleep and autonomic issues [10].

Iron chelators and antioxidants therefore hold promise as potential neuroprotective therapies for PD. Ward et al [11] were the first group to demonstrate that bidentate iron chelators like deferiprone (DFP) and deferoxamine (DFO) were able to remove excess brain iron in animal models of brain iron overload. Iron chelators have since been demonstrated to be neuroprotective in 6-hydroxydopamine (6-OHDA) animal models of PD $[12,13]$. Furthermore, a recent clinical trial of the iron chelator DFP correlated chelator use with a decrease in the UPDRS score for motor symptoms [14]. Similarly, in both in vitro and in vivo PD models, curcumin has been able to protect against dopaminergic neurodegeneration. For example, in a cellular rotenone-induced neurotoxin model of PD curcumin reduced 
reactive oxygen species (ROS) and cytotoxicity as well as inhibited caspase 3 and 9 activation, preventing apoptosis [15]. Likewise, in a mutant A53T $\alpha$-synuclein ( $\alpha$-syn) cellular model of PD curcumin pre-treatment reduced cell death as well as levels of mutant a-syn [16] and in an in vivo drosophila model of PD curcumin not only reduced cell death but also ameliorated PD-like symptoms [15]. More recent evidence suggests that curcumin is also protective against mitochondrial dysfunction. Van der Merwe et al [17] showed curcumin to be protective in a PINK1 knock down model of PD and PINK1 has an intrinsic role in the removal of dysfunctional mitochondria with mutations in PINK1 resulting in an early-onset autosomal recessive PD. The combination of iron chelators and antioxidant molecules may present a promising potential therapeutic approach for PD as together they are able to completely restore brain function impaired by iron overload in animal models [18]

The free radical scavenging properties of curcumin and the potential of iron chelators such as DFO to limit the availability of detrimental free iron may be a promising mechanism to limit the degenerative process in PD. That said, the therapeutic value of these two compounds is limited as curcumin is unstable with low bioavailability and both are unlikely to access the brain at therapeutic concentrations $[19,20]$. Furthermore, long-term effects of continuous iron chelator use are unknown in PD and due to the non-specific nature of such chelators the side effects could be serious since iron is an essential part of numerous cellular processes such as respiration [21] and neurotransmitter synthesis [22, 23].

Nanocarriers have demonstrated potential as targeted delivery systems to improve stability of labile molecules and enhance delivery across membranes, including the blood-brain barrier (BBB), whilst retaining the potency of entrapped molecules [24, 25]. For example, studies which have altered the formulation of curcumin such as encapsulation in glycerol 
monooleate have increased the half-life of curcumin [26]. Other nanoformulations of curcumin such as the poly(lactic-co-glycolic acid) (PLGA) nanoformulation have demonstrated BBB penetration and the ability to and enter brain tissues including the cerebral cortex and the hippocampus however, they are also found in other organs such as the spleen and the liver [27]. Zupančič et al [25] developed a DQAsome nanoformulation of curcumin using vesicles formed from the amphiphile dequalinium (DQA) which were able to specifically target mitochondria. Mitochondrial targeting of antioxidants in PD would be highly beneficial since mitochondria are the main site of intracellular free radical formation $[7,8,28]$. However, such nanoformulations of curcumin were not developed to gain access into the brain or treat neurodegeneration and therefore need to be assessed in relevant models in order to evaluate the potential benefit for PD. Therefore, this study aimed to develop nanocarrier, nasal delivery systems for curcumin and/or DFO using the amphiphilic polymer, Pluronic F68 (P68), with or without DQA to protect against rotenone-induced changes in cell viability and oxidative stress in SH-SY5Y neuronal cells.

\section{Materials and Methods}

\subsection{Materials}

Unless otherwise stated, all chemicals were analytical grade. Dequalinium chloride hydrate (DQA; 95\%), Dulbecco's phosphate buffered saline (PBS), Curcumin from Curcuma longa (Turmeric), powder ( $\geq 80 \%)$, Deferoxamine mesylate salt (92.5\%), Protease inhibitor cocktail (PIC), Thiazolyl Blue Tetrazolium Blue (MTT), Dimethyl sulfoxide (DMSO), 2',7'Dichlorofluorescin diacetate (DCFH-DA) and 2,2'-Azobis(2-methylpropionamidine) dihydrochloride (ABAP) were purchased from Sigma-Aldrich, U.K. Methanol (HPLC grade), Lglutamine, fetal bovine serum (FBS), Dulbecco's Modified Eagle Medium (DMEM) 


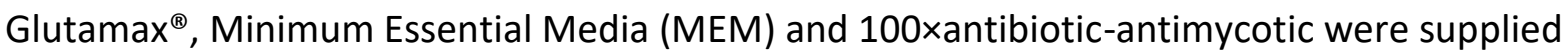
by Fisher Scientific, U.K. The TBARS Parameter Assay Kit was purchased from R\&D Systems, Parameter TM, UK. The BCA protein assay kit was from Pierce (Thermo Fisher Scientific, UK). SH-SY5Y cells were purchased from the American Type Culture Collection (ATCC CRL-2266, USA). Flasks were from Nunc, Denmark and culture plates from Corning, UK.

\subsection{Development and characterisation of nanoformulations}

\subsection{Preparation of Curcumin and/or deferoxamine nanoformulations}

All nanoformulations were prepared using a modified thin-film hydration method $[25,29]$. Briefly, P68 nanocarriers with or without DQA, curcumin and/or deferoxamine (DFO) at different ratios (table I) were formulated using a rotary evaporator (Hei-VAP Advantage Rotary Evaporator, Heidolph, Germany) at $200 \mathrm{rpm}$ and $80^{\circ} \mathrm{C}$ under vacuum, using methanol as the solvent. The resultant thin film was hydrated with $10 \mathrm{ml}$ of distilled water and mixed thoroughly at $80^{\circ} \mathrm{C}$ for $1-2$ min and sonicated using a VWR Ultrasonic cleaner bath USC300T (VWR International Limited, U.K.) for a further 1 min until the film was fully removed and dissolved in the water. In order to remove any unloaded curcumin and/or DFO, the obtained formulation was filtered through a sterile $0.22 \mu \mathrm{m}$ filter (Millex-MP, Millipore, Carrigtwohill, Ireland). In order to store samples for further analysis, some samples were freeze dried (lyophilized) using a Virtis AdVantage 2.0 BenchTop freezedryer (SP Industries, UK).

\subsection{Size and Surface Charge of the nanoformulations}

The size and surface charge of prepared nanoformulations were measured following filtration using the Zetasizer Nano ZS (Malvern Instruments, U.K.). Size distribution was measured via photon correlation spectroscopy as Z-Ave hydrodynamic diameter and polydispersity index (PDI). Transmission electronmicroscopy (TEM) analysis was performed 
to analyse morphology (size and shape) of the nanoformulation complexes. The surface charge was measured by laser Doppler micro-electrophoresis. All experiments were performed in triplicate.

\subsection{Determination of Drug Loading and Encapsulation Efficiency}

UV-Visible (UV-Vis) spectroscopy was employed to study drug loading and encapsulation efficiency of the nanoformulations based on the calibration curves of free curcumin and DFO. Methanol and water were added in a 1:1 ratio to dissolve the carrier in order to release the drug and achieve the theoretical concentration of $10 \mu \mathrm{g} / \mathrm{ml}$ curcumin or $20 \mu \mathrm{g} / \mathrm{ml}$ DFO. Curcumin and DFO content was calculated using UV-vis spectroscopy at $423 \mathrm{~nm}$ and $204 \mathrm{~nm}$ respectively. The percentage of drug loading and encapsulation efficiency were calculated using the following equations:

- $\quad$ Drug loading $(\%)=$ (determined mass of drug within nanocarriers / mass of drugloaded nanocarriers) X 100

- Encapsulation efficiency $(\%)=$ (determined mass of drug within nanocarriers / theoretical mass of drug within nanocarriers) $\times 100$

\subsection{Assessing the therapeutic potential of nanoformulations in a model of PD}

\subsection{Cell culture and lysate harvesting}

The human neuroblastoma SH-SY5Y cell line was used to create and in vitro model of PD (reviewed in Xicoy et al. [30]). SH-SY5Y cells were grown in DMEM - Glutamax ${ }^{\circledR}, \mathrm{pH} 7.4$ supplemented with $10 \% \mathrm{FBS}$ and $1 \%$ antibiotic/antimycotic in a 5\% CO2 environment at $37^{\circ} \mathrm{C}$. SH-SY5Y cells were thawed and left to grow in plastic T75 $\left(75 \mathrm{~cm}^{2}\right)$ flasks until they reached $70 \%$ confluence. Adherent cells were then detached from the surface of the flasks 
via trypsinisation, counted and seeded into well plates at specific numbers according to the bioassay being performed (6-well or 96-well plates). To produce a model of PD, SH-SY5Y cells were treated with $100 \mu \mathrm{M}$ rotenone for $24 \mathrm{~h}$ in order to reduce cell viability by approximately $50 \%$ (supplementary figure 1 ).

\subsection{Cell viability - MTT Assay}

The protective properties of drug-loaded nanocarriers against rotenone-induced reduction in cell viability was assessed using the MTT Assay. Briefly, SH-SY5Y cells grown at a seeding density of $1,000,000$ cells $/ \mathrm{cm}^{2}$ in 96 well plates until confluent. The cells were pre-treated for $3 \mathrm{~h}$ with either free or nanoformulated curcumin (5 and $10 \mu \mathrm{M})$, DFO $(100 \mu \mathrm{M})$ or combined curcumin $(5$ or $10 \mu \mathrm{M})$ and DFO $(50$ or $100 \mu \mathrm{M})$, or with corresponding unloaded, blank formulations. SH-SY5Y cells were then treated with $100 \mu \mathrm{M}$ rotenone for $24 \mathrm{~h}$, each well containing a total volume of $200 \mu$ l. The cells were then incubated with an additional $20 \mu \mathrm{l}$ of $5 \mathrm{mg} / \mathrm{ml}$ solution of MTT DPBS solution for $4 \mathrm{~h}$ at $37^{\circ} \mathrm{C}$. Following aspiration, $100 \mu \mathrm{l}$ of DMSO was added to each well to dissolve the formazan crystals. To ensure DMSO was mixed well, plates were placed on a shaker at 75rpm for $15 \mathrm{~min}$ and the absorbance was read at $570 \mathrm{~nm}$ on a spectrophotometer.

\subsection{Lipid Peroxidation - TBARS assay}

The thiobarbituric acid-reactive-substances (TBARS) assay was used to assess ROS generated oxidative stress, specifically lipid peroxidation [31, 32]. Briefly, SH-SY5Y cells were grown in 6-well plates until confluent. Following pre-treatment with the relevant free or nanoformulated curcumin and/or DFO, the cells were incubated with $100 \mu \mathrm{M}$ rotenone for 24h. Cells were then washed once with DPBS and lysed at $4^{\circ} \mathrm{C}$ as previously described 
by Zariwala et al [33], using $350 \mu$ ice-cold lysis buffer $(50 \mathrm{mM} \mathrm{NaOH}$ supplemented with $1 \mu \mathrm{g} / \mathrm{ml}$ protease inhibitor cocktail - PIC) whilst rocking gently for $40 \mathrm{~min}$ in ice trays on a plate shaker ( $8 \mathrm{rpm})$. Cell lysates were then collected using sterile cell scrapers and aliquoted into microcentrifuge tubes ready for further analysis. The lysate total protein concentrations were determined using the Pierce BCA kit with BSA as standard. The TBARS assay was carried out in accordance to manufacturer guidelines (R\&D Systems, Parameter TM). Briefly, freshly prepared TBA was added to TBARS acid-treated cell lysate samples (loaded in triplicate) and incubated at $60{ }^{\circ} \mathrm{C}$ for $2.5 \mathrm{~h}$. The samples were read at absorbance $532 \mathrm{~nm}$ before and after incubation to estimate the formation of TBARS.

\subsection{Cellular antioxidant activity - CAA Assay}

The cellular antioxidant activity was measured using the method described by Chen et al [34] which is a slightly modified version of the assay developed by Wolfe, et al [35] and Hu, et al [36]. SH-SY5Y cells were seeded in black-walled, clear-bottom 96-well microplates. Once confluent, cells were washed with DPBS and treated with different concentrations of $200 \mu$ l of drug-loaded nanocarriers, free drug solution or MEM only (control) for $1-3 \mathrm{~h}$ at $37^{\circ} \mathrm{C}$. Cells were then washed with MEM and treated with $200 \mu \mathrm{l}$ of $100 \mu \mathrm{M}$ DCFH-DA (dissolved in MEM) and incubated for a further $30 \mathrm{~min}$ at $37^{\circ} \mathrm{C}$. Following aspiration, each well was treated with $100 \mu$ l of $600 \mu \mathrm{M}$ ABAP (dissolved in MEM). The fluorescence of the cells in the 96-well plate was read every 5 minutes for $1 \mathrm{~h}$ at $528 \mathrm{~nm}$ and $485 \mathrm{~nm}$ emission and excitation wavelengths (respectively).

\subsection{Statistical Analysis.}

For all experiments the mean of six replicates was calculated for each treatment, and the data expressed as mean \pm standard deviation (S.D.). The MTT and TBARS assay results were 
statistically analysed using one-way analysis of variance (ANOVA) followed by Dunnett's T3

post hoc test. A two-way ANOVA followed by Tukey's multiple comparisons post hoc test was used to analyse the CAA assay results (PRISM software package, Version 7, Graphpad Software Inc., San Diego, USA).

\section{Results}

All P68 nanoformulations demonstrated high encapsulation efficiency (66-95\%), DFOloaded P68 nanocarriers had the highest mean encapsulation efficiency (95\%), curcuminloaded nanocarriers had $79 \%$ and the combined curcumin (67\%) and DFO (66\%) nanocarriers had the lowest encapsulation efficiencies (table 1). The mean P68 nanocarrier size was $<200 \mathrm{~nm}$ in all cases, however curcumin-loaded nanocarriers had the largest particle sizes (table 1). All P68 nanoformulations had low polydispersity as represented by mean polydispersity indices $<0.22$ suggesting that the majority of the nanocarriers within the formulation are of a similar size (table 1). All P68 nanocarriers had similar low negative surface charges (table 1). The addition of DQA to formulate the P68+DQA nanocarriers did not significantly change the particle size, however it did result in more positive surface charges (0.02 to 9.29) and in all cases appears to have increased encapsulation efficiency; curcumin-loaded nanocarrier encapsulation efficiency increased by $7 \%$, DFO-loaded nanocarriers increased by $1 \%$ and combined curcumin and DFO-loaded nanocarriers had the largest increase of $10-20 \%$ for each drug, from $67 \%$ and $66 \%$ to $82 \%$ and $96 \%$, respectively (table 1).

$24 \mathrm{~h}$ treatment with $100 \mu \mathrm{M}$ rotenone significantly reduced SH-SY5Y cell viability by $40-50 \%$ in all experiments (figure $2 A, B$, supplementary figure 1 ). Cytotoxicity was not observed for the tested concentrations of any nanoformulation $\geq 80 \%(n=6)$. When comparing the effects 
of free drug and $\mathrm{P} 68$ nanocarriers on cell viability following rotenone treatment, a significant difference in mean cell viability was observed between the different treatments $(F(16,94)=93.97, p<0.0001)$ (figure $2 A) .3$-hour pre-treatment of P68 nanocarriers of $5 \mu M$ and $10 \mu \mathrm{M}$ curcumin $(p=0.0039$ and $p=0.0078$, respectively), 100 $\mu \mathrm{M}$ DFO $(p=0.0002)$ and combined $5 \mu \mathrm{M}$ curcumin and $50 \mu \mathrm{M}$ DFO $(p=0.0029)$ significantly protected against the reduction in cell viability induced by $24 \mathrm{~h}$ treatment with $100 \mu \mathrm{M}$ rotenone (figure $2 \mathrm{~A}$ ). Of the P68 nanoformulation conditions which were protective, no single condition was significantly more protective than another. None of the free drug pre-treatment conditions apart from combined $5 \mu \mathrm{M}$ curcumin and $50 \mu \mathrm{M}$ DFO $(p=0.0219)$ were able to significantly protect against rotenone and pre-treatment with the combination of free curcumin $(10 \mu \mathrm{M})$ and free DFO $(100 \mu \mathrm{M})$ followed by rotenone treatment appears to be more toxic than rotenone alone ( $p=0.0003$ ) (figure $2 A)$. There was no significant difference in cell viability following pre-treatment with P68 nanocarriers compared to the corresponding free drug pretreatments apart from with $10 \mu \mathrm{M}$ curcumin $+100 \mu \mathrm{M}$ DFO where pre-treatment with P68 nanocarriers resulted in higher cell viability $(p=0.0032)$.

Similarly, there was a significant difference in mean cell viability between the different treatments when comparing free drug and P68+DQA nanocarrier pre-treatments prior to rotenone treatment $(F(16,60)=5.639, p<0.0001)$ (figure $2 B)$. The majority of P68+DQA nanoformulation pre-treatments were able to protect against rotenone, $(5 \mu \mathrm{M}$ curcumin $(p<$ $0.0001), 10 \mu \mathrm{M}$ curcumin $(p=0.0331), 5 \mu \mathrm{M}$ curcumin $+50 \mu \mathrm{M}$ DFO $(p=0.0104), 10 \mu \mathrm{M}$ curcumin $+100 \mu \mathrm{M}$ DFO $(p=0.014))$, including the combination for $10 \mu \mathrm{M}$ curcumin and $100 \mu \mathrm{M}$ DFO which was not protective in the P68 or free conditions (figure $2 \mathrm{~B}$ ). However, P68+DQA nanocarriers of $100 \mu \mathrm{M}$ DFO did not significantly protect against rotenone. In these results, free $5 \mu \mathrm{M}$ curcumin pre-treatment was also able to significantly protect 
against rotenone $(p=0.0455)$. Similar to when using P68 nanocarriers, when comparing P68+DQA nanocarriers with the corresponding free drug pre-treatments, there was only a significant difference found for $10 \mu \mathrm{M}$ curcumin $+100 \mu \mathrm{M}$ DFO where pre-treatment with P68+DQA nanocarriers resulted in higher cell viability $(p=0.004)$.

A significant difference in mean TBARS concentrations was observed between the different treatments when testing both $\mathrm{P} 68(\mathrm{~F}(16,84)=35.36, \mathrm{p}<0.0001)$ and $\mathrm{P} 68+\mathrm{DQA}(F(16,17)=$ $41.55, p<0.0001$ ) nanocarriers compared to the corresponding free drugs (figure 3A, B). 3hour pre-treatment of all preparations (free drug, P68 and P68+DQA nanoformulations) of all conditions $(5 \mu \mathrm{M}$ curcumin, $10 \mu \mathrm{M}$ curcumin, $100 \mu \mathrm{M}$ DFO, $5 \mu \mathrm{M}$ curcumin $+50 \mu \mathrm{M}$ DFO, $10 \mu \mathrm{M}$ curcumin $+100 \mu \mathrm{M}$ DFO) significantly protected against rotenone-induced lipid peroxidation, measured by TBARS concentration (figure 3A, 4B). 3-hour pre-treatment with P68 nanocarriers of $5 \mu \mathrm{M}$ and $10 \mu \mathrm{M}$ curcumin ( $p=0.0012$ and $p=0.0135$, respectively), $100 \mu \mathrm{M}$ DFO $(p=0.0012)$ and the combination of $5 \mu \mathrm{M}$ curcumin and $50 \mu \mathrm{M}$ DFO $(p=0.0007)$ were significantly more protective against rotenone-induced increase in TBARS concentration compared to the corresponding free drug conditions (figure $3 \mathrm{~A}$ ). There was no significant difference in the ability of P68+DQA nanocarriers and the corresponding free drug pre-treatments to protect against rotenone-induced lipid peroxidation (figure 3B).

The CAA results showed significant differences in cellular antioxidant activity between the different treatment preparation types $(F(4,100)=27.78, p<0.0001)$ and the different concentrations of treatments $(F(3,100)=26.78, p=p<0.0001)$ (figure $4 A, B)$. P68+DQA drug loaded nanocarriers had significantly higher antioxidant capability than P68 nanocarriers in all corresponding conditions except $100 \mu \mathrm{M}$ DFO where there was no significant difference (figure 4A). The highest difference in antioxidant activity between 
these formulations was at $10 \mu \mathrm{M}$ curcumin concentrations, where P68+DQA curcuminloaded nanocarriers had $63 \%$ higher antioxidant activity compared to P68 curcumin-loaded nanocarriers $(p<0.0001)$ (figure 4A). Likewise, P68+DQA drug loaded nanocarriers had significantly higher cellular antioxidant activity compared to P68 drug loaded nanocarriers at $5 \mu \mathrm{M}$ curcumin $(48 \%, p=0.001), 5 \mu \mathrm{M}$ curcumin $+50 \mu \mathrm{M}$ DFO $(48 \%, p=0.0012), 10 \mu \mathrm{M}$ curcumin $+100 \mu \mathrm{M}$ DFO $(30 \%, p=0.0063)$ concentrations. $100 \mu \mathrm{M}$ DFO P68+DQA nanocarriers also had higher antioxidant activity, 21\% higher than P68 DFO-loaded nanocarriers at this concentration, although not significant (figure 4A). P68+DQA nanocarriers were also significantly superior in cellular antioxidant activity compared to the corresponding curcumin free drug+DQA conditions $(5 \mu \mathrm{M}$ and $10 \mu \mathrm{M}$ curcumin $(p=0.0003$ and $p=0.0022$ respectively) (figure $4 \mathrm{~A}$ ). There was no significant difference in cellular antioxidant activity of P68+DQA nanocarriers compared to any of the corresponding free drug alone conditions. There was also no significant difference between P68 nanocarriers and free drug+DQA for any of the conditions apart from $10 \mu \mathrm{M}$ curcumin $+100 \mu \mathrm{M}$ DFO where free drug+DQA was superior $(p=0.0337$ ) (figure $4 A$ ). However, in the majority of cases the free drug conditions had a significantly higher cellular antioxidant unit than the corresponding P68 nanocarriers (10 MM curcumin $(p=0.0083), 100 \mu \mathrm{M}$ DFO ( $p=0.0343)$, combination of curcumin and DFO for both $5 \mu \mathrm{M}+50 \mu \mathrm{M}(p=0.0203)$ and $10 \mu \mathrm{M}+100 \mu \mathrm{M}(p$ $=0.0082$ ), respectively) (figure 4A). In all preparations, free drug, free drug+DQA, P68 and P68+DQA, the combination of $10 \mu \mathrm{M}$ curcumin and $100 \mu \mathrm{M}$ DFO had the most antioxidant potential compared to the majority of other conditions (figure 4B).

\section{Discussion}


There is much evidence suggesting that curcumin and DFO are protective in numerous models of PD [12-17] and that the combination of such antioxidants and iron chelators may have even more potent effects [18]. However, the potential of these compounds as possible therapies for PD has been limited due to numerous factors such as brain penetrance, bioavailability and stability $[19,20]$. Advances in nanotechnology have provided a possible solution to these issues as nanocarriers can be developed to retain the potency of the entrapped molecule while enhancing targeted delivery and passage across biological membranes $[24,25,37,38]$. The aim of this study was to develop nanocarrier, nasal delivery systems for curcumin and DFO as well as the combination of the two and assess the ability of such nanocarriers to protect against reduced cell viability and increased oxidative stress in a cellular rotenone model of PD.

Nanocarriers were developed using the amphiphilic polymer P68 as it has been successfully used to develop micellar nanocarriers of numerous compounds [39-41] including curcumin for other indications [42-45]. Mitochondrial targeting of nanocarriers using dequilinium has been established $[25,29,46]$ therefore, the addition of dequilinium to the nanoformulation was used to assess whether mitochondrial targeting would result in increased potency. Curcumin and DFO were successfully incorporated into both P68 and P68+DQA nanocarriers (table 1). The mean size of both P68 and P68+DQA nanocarriers was fairly consistent, in both cases curcumin-loaded nanocarriers had a larger mean size than DFO-loaded nanocarriers, and the addition of DFO to curcumin further increased nanocarrier size but all were under $200 \mathrm{~nm}$. Such results are consistent with the ability to cross the BBB $[37,47]$. The mean surface charge of the P68 nanocarriers was relatively neutral, between -0.44 and $5.88 \mathrm{mV}$ (table 1). This may also be beneficial for brain penetrance because most 
nanocarriers with a low to moderate surface charge between $-1 m V$ and $-15 m V$ have been shown to pass the BBB [48-51]. The addition of DQA to the formulations resulted in a moderate increase in charge to a more positive state $(+0.02$ to $+9.29 \mathrm{mV})$, consistent with previous use of DQA in lipid-based formulations [25], however to a much lesser extent as such studies reported charges up to $+50 \mathrm{mV}$. This only slight - moderate increase in charge is more suitable for brain delivery as overall such charges are relatively neutral, not so positive to cause toxicity to the BBB, known to occur at high positive charges [52], but a large enough shift in charge to potentially improve physical stability [25]. Further to this, drugs are more likely to be able to pass the BBB if they are not rapidly cleared from the bloodstream [38] and neutral nanocarriers have been shown to have longer circulation time in the bloodstream compared to those that are very negatively or very positively charged [53].

P68 and P68+DQA nanocarriers were protective against rotenone-induced reductions in cell viability suggesting that they may be able to protect against cell death induced by rotenone. It was expected that the combination of the highest tolerable concentrations of curcumin and DFO would be the most effective at protecting against rotenone due to the combination of reduced free iron and increased antioxidants which together should prevent the accumulation of toxic free radicals which drive oxidative stress. However, pre-treatment with the combination of these free drugs appeared to result in toxicity following rotenone treatment (figure $2 \mathrm{~A}, \mathrm{~B}$ ). That said, following the combined formulation of $10 \mu \mathrm{M}$ curcumin and $100 \mu \mathrm{M}$ DFO into P68 and P68+DQA nanocarriers, pre-treatment with the combined drugs not only removed the toxicity previously observed but also significantly increased cell viability (figure $2 \mathrm{~A}, \mathrm{~B}$ ). P68 nanocarriers of $10 \mu \mathrm{M}$ curcumin and $5 \mu \mathrm{M}$ curcumin $+50 \mu \mathrm{M}$ DFO were the most successful at protecting cell viability against rotenone compared to the other 
drug-loaded P68 nanocarriers, retaining cell viability over $80 \%$ (figure $2 \mathrm{~A}$ ). Whereas, increasing concentrations of P68+DQA curcumin and combined curcumin + DFO nanocarriers increasingly protected cell viability, with the pre-treatment of $10 \mu \mathrm{M}$ curcumin $+100 \mu \mathrm{M}$ DFO retaining $90 \%$ cell viability (figure $2 \mathrm{~B}$ ). Although DFO-loaded P68+DQA nanocarriers did not significantly protect cell viability against rotenone, the addition of $50 \mu \mathrm{M}$ and $100 \mu \mathrm{M}$ DFO to $5 \mu \mathrm{M}$ and $10 \mu \mathrm{M}$ curcumin-loaded P68+DQA nanocarriers resulted in a modest increase in protection, by $10 \%$ and $11 \%$ respectively.

Similarly, all P68 and P68+DQA drug-loaded nanocarriers protected against rotenoneinduced lipid peroxidation, in all cases maintaining TBARS levels equivalent to control conditions (figure $3 \mathrm{~A}, \mathrm{~B}$ ). The results suggest that $\mathrm{P} 68$ but not P68+DQA drug-loaded nanocarriers are significantly more protective than the corresponding free drug pretreatments (figure 3A, B). However, it appears that the free drug preparation of all conditions resulted in lower TBARS concentrations in the experiment using the P68+DQA nanocarriers compared to when using P68 nanocarriers. This could be due to the cells having a higher starting TBARS concentration in the P68 experiment as measured by the control cells which did not receive any pre-treatment or rotenone treatment.

The cellular antioxidant activity results suggest that P68+DQA drug-loaded nanocarriers of all conditions have more potent antioxidant potential than P68 drug-loaded nanocarriers. The addition of DQA to the formulations resulted in a significant increase from $48 \%$ to $65 \%$ in cellular antioxidant activity in all conditions apart from 100uM DFO where $20 \%$ higher antioxidant activity was observed but did not reach statistical significance (figure 4A). This suggests that the addition of DQA, which is known to target mitochrondria $[25,29,46]$, significantly improves the antioxidant ability of the formulations. This result was anticipated 
as mitochondria are the main site of intracellular free radical formation $[7,8,28]$.

Furthermore, in nearly all preparations, the combination of $10 \mu \mathrm{M}$ curcumin and $100 \mu \mathrm{M}$ DFO had the most antioxidant activity (figure 4B), suggesting that the combination of increasing antioxidants and decreasing free iron may be the most successful strategy for reducing ROS production.

Overall, these results suggest that all drug-loaded nanocarriers were at least as capable as the corresponding free drug conditions at protecting against reduced cell viability and increased lipid peroxidation induced by rotenone. As the potential therapeutic value of the free curcumin and DFO is limited due to issues such as bioavailability and brain penetrance $[19,20]$, the nanocarrier delivery systems may provide a viable solution as the particle size and charge data indicated that these formulations would be able to pass the BBB. Moreover, the ability to target these non-specific drugs to mitochrondria using P68+DQA nanocarriers seemed to result in increased cellular antioxidant activity. The outcome measures of these experiments focused mostly on antioxidant capability therefore it is unsurprising that the curcumin formulations were generally superior to DFO as curcumin is known to obtain antioxidant properties. However, for most preparations the addition of DFO to curcumin resulted in increased cellular antioxidant activity suggesting that a combination therapy may provide a good approach to combat oxidative stress in PD models. This is another benefit of using nanocarriers as, unlike combination delivery of free drugs which is challenging due to the distinct pharmacokinetic profiles of each drug, nanocarriers can ensure that drugs are delivered together for synergistic treatment [54].

In summary, this study demonstrates for the first time the formulation and delivery of P68 and P68+DQA curcumin and/or DFO nanocarriers to protect against oxidative stress induced 
by a cellular rotenone model of PD. This strategy may thus provide a novel approach to fully utilise their therapeutic benefit for PD. The next steps will be to assess additional parameters such as iron, dopamine and alpha synuclein prior to and following pretreatment with these formulations. As these results are from a neurotoxin model, in a neuroblastoma cell line that do not only contain dopaminergic neurons, further experiments will be carried out to determine if the effects are seen in more accurate models of PD, for example using patient derived induced pluripotent stem cells and in vivo alpha synuclein models. Such additional data would be valuable in providing the evidence needed as to whether these formulations can move forward into clinical testing as potential diseasemodifying therapies for PD.

\section{Acknowledgments}

Leah Mursaleen was supported by the FST studentship grant, University of Westminster.

The authors wish to thank Andrew Weston and Stefanie Chan from University College London for their assistance in characterising the nanocarriers.

\section{Conflicts of Interest}

The authors have no conflict of interest to report. 


\section{References}

[1] Dexter DT, Wells FR, Agid F, Agid Y, Lees AJ, Jenner P, Marsden CD (1987) Increased nigral iron content in postmortem parkinsonian brain. The Lancet 330, 1219-1220.

[2] Griffiths PD, Dobson BR, Jones GR, \& Clarke DT (1999) Iron in the basal ganglia in Parkinson's disease: An in vitro study using extended X-ray absorption fine structure and cryo-electron microscopy. Brain 122, 667-673.

[3] Graham JM, Paley MN, Grünewald RA, Hoggard N, Griffiths PD (2000) Brain iron deposition in Parkinson's disease imaged using the PRIME magnetic resonance sequence. Brain 123, 2423-2431.

[4] Martin WRW, Wieler M, Gee M (2008) Midbrain iron content in early Parkinson disease A potential biomarker of disease status. Neurology 70, 1411-1417.

[5] Wallis LI, Paley MN, Graham JM, Grünewald RA, Wignall EL, Joy HM, Griffiths PD (2008) MRI assessment of basal ganglia iron deposition in Parkinson's disease. Journal of Magnetic Resonance Imaging 28, 1061-1067.

[6] Rossi M, Ruottinen H, Soimakallio S, Elovaara I, Dastidar P (2013) Clinical MRI for iron detection in Parkinson's disease. Clinical imaging 37, 631-636.

[7] Kandola K, Bowman A, Birch-Machin MA (2015) Oxidative stress-a key emerging impact factor in health, ageing, lifestyle and aesthetics. International journal of cosmetic science, 37, 1-8.

[8] Costa-Mallen P, Gatenby C, Friend S, Maravilla KR, Hu SC, Cain KC, Anzai Y (2017) Brain iron concentrations in regions of interest and relation with serum iron levels in Parkinson disease. Journal of the neurological sciences 378, 38-44.

[9] Ben-Shachar D, Youdim MBH (1991) Intranigral iron injection induces behavioral and biochemical "parkinsonism" in rats. Journal of neurochemistry 57, 2133-2135.

[10] Liu Z, Shen HC, Lian TH, Mao L, Tang SX, Sun L, Zuo LJ (2017) Iron deposition in substantia nigra: abnormal iron metabolism, neuroinflammatory mechanism and clinical relevance. Scientific reports 7, 14973.

[11] Ward RJ, Dexter D, Florence A, Aouad F, Hider R, Jenner P, Crichton RR (1995) Brain iron in the ferrocene-loaded rat: its chelation and influence on dopamine metabolism. Biochemical pharmacology 49, 1821-1826.

[12] Youdim MB, Stephenson G, Shachar DB (2004) Ironing iron out in Parkinson's disease and other neurodegenerative diseases with iron chelators: a lesson from 6hydroxydopamine and iron chelators, desferal and VK-28. Annals of the New York Academy of Sciences 1012, 306-325. 
[13] Dexter DT, Statton SA, Whitmore C, Freinbichler W, Weinberger P, Tipton KF, Della Corte L, Ward RJ, Crichton RR (2011) Clinically available iron chelators induce neuroprotection in the 6-OHDA model of Parkinson's disease after peripheral administration. Journal of neural transmission 118, 223-231.

[14] Devos D, Moreau C, Devedjian JC, Kluza J, Petrault M, Laloux C, Duhamel A (2014) Targeting chelatable iron as a therapeutic modality in Parkinson's disease. Antioxidants \& redox signaling $21,195-210$.

[15] Liu Z, Li T, Yang D, Smith WW (2013) Curcumin protects against rotenone-induced neurotoxicity in cell and drosophila models of Parkinson's disease. Advances in Parkinson'S Disease 2, 18.

[16] Jiang TF, Zhang YJ, Zhou HY, Wang HM, Tian LP, Liu J, Chen SD (2013) Curcumin ameliorates the neurodegenerative pathology in A53T $\alpha$-synuclein cell model of Parkinson's disease through the downregulation of mTOR/p70S6K signaling and the recovery of macroautophagy. Journal of Neuroimmune Pharmacology 8, 356-369.

[17] Van der Merwe C, Van Dyk HC, Engelbrecht L, van der Westhuizen FH, Kinnear C, Loos B, Bardien S (2017) Curcumin rescues a PINK1 knock down SH-SY5Y cellular model of Parkinson's disease from mitochondrial dysfunction and cell death. Molecular neurobiology 54, 2752-2762.

[18] Sripetchwandee J, Pipatpiboon N, Chattipakorn N, Chattipakorn S (2014) Combined therapy of iron chelator and antioxidant completely restores brain dysfunction induced by iron toxicity. PLoS One 9, p.e85115.

[19] Yang KY, Lin LC, Tseng TY, Wang SC, Tsai TH (2007) Oral bioavailability of curcumin in rat and the herbal analysis from Curcuma longa by LC-MS/MS. Journal of chromatography B 853, 183-189.

[20] Martin-Bastida A, Ward RJ, Newbould R, Piccini P, Sharp D, Kabba C, Crichton RR (2017) Brain iron chelation by deferiprone in a phase 2 randomised doubleblinded placebo controlled clinical trial in Parkinson's disease. Scientific reports 7, 1398.

[21] Pino J, da Luz MH, Antunes HK, Giampá SQ, Martins VR, Lee KS (2017) IronRestricted Diet Affects Brain Ferritin Levels, Dopamine Metabolism and Cellular Prion Protein in a Region-Specific Manner. Frontiers in molecular neuroscience 10, 145.

[22] Youdim MBH, Green AR (1978) Iron deficiency and neurotransmitter synthesis and function. Proceedings of the Nutrition Society 37, 173-179.

[23] Hidalgo C. Núñez MT (2007) Calcium, iron and neuronal function. IUBMB life 59, 280-285.

[24] Masserini M (2013) Nanoparticles for brain drug delivery. ISRN biochemistry 2013. 1-18. 
[25] Zupančič S, Kocbek P, Zariwala MG, Renshaw D, Gul MO, Elsaid Z, Somavarapu S (2014) Design and development of novel mitochondrial targeted nanocarriers, DQAsomes for curcumin inhalation. Molecular pharmaceutics 11, 2334-2345.

[26] Mohanty C, Sahoo SK (2010) The in vitro stability and in vivo pharmacokinetics of curcumin prepared as an aqueous nanoparticulate formulation. Biomaterials 31, 65976611.

[27] Tsai YM, Chien CF, Lin LC, Tsai TH (2011) Curcumin and its nano-formulation: the kinetics of tissue distribution and blood-brain barrier penetration. International journal of pharmaceutics $416,331-338$.

[28] Thomas C, Mackey MM, Diaz AA, Cox DP (2009) Hydroxyl radical is produced via the Fenton reaction in submitochondrial particles under oxidative stress: implications for diseases associated with iron accumulation. Redox Report 14, 102-108.

[29] Weissig V, Lasch J, Erdos G, Meyer HW, Rowe TC, Hughes J (1998) DQAsomes: a novel potential drug and gene delivery system made from Dequalinium. Pharm Res 15, 334-7.

[30] Xicoy H, Wieringa B, Martens GJ (2017) The SH-SY5Y cell line in Parkinson's disease research: a systematic review. Molecular neurodegeneration 12, 10.

[31] Dutta RK, Nenavathu BP, Gangishetty MK, Reddy AVR (2012) Studies on antibacterial activity of $\mathrm{ZnO}$ nanoparticles by ROS induced lipid peroxidation. Colloids and Surfaces B: Biointerfaces 94, 143-150.

[32] Chakraborti S, Chakraborty S, Saha S, Manna A, Banerjee S, Adhikary A, Chakrabarti $P$ (2017) PEG-functionalized zinc oxide nanoparticles induce apoptosis in breast cancer cells through reactive oxygen species-dependent impairment of DNA damage repair enzyme NEIL2. Free Radical Biology and Medicine 103, 35-47.

[33] Zariwala MG, Somavarapu S, Farnaud S, Renshaw D (2013) Comparison study of oral iron preparations using a human intestinal model. Scientia pharmaceutica 81, 1123.

[34] Chen X, Zou LQ, Niu J, Liu W, Peng SF, Liu CM (2015) The stability, sustained release and cellular antioxidant activity of curcumin nanoliposomes. Molecules 20, 1429314311.

[35] Wolfe KL, Liu RH (2007) Cellular antioxidant activity (CAA) assay for assessing antioxidants, foods, and dietary supplements. Journal of agricultural and food chemistry $55,8896-8907$.

[36] Hu B, Ting Y, Zeng X, Huang Q (2013) Bioactive peptides/chitosan nanoparticles enhance cellular antioxidant activity of (-)-epigallocatechin-3-gallate. Journal of agricultural and food chemistry $61,875-881$. 
[37] Grabrucker AM, Ruozi B, Belletti D, Pederzoli F, Forni F, Vandelli MA, Tosi G (2016) Nanoparticle transport across the blood brain barrier. Tissue barriers 4, e1153568.

[38] Saraiva C, Praça C, Ferreira R, Santos T, Ferreira L, Bernardino L (2016) Nanoparticle-mediated brain drug delivery: overcoming blood-brain barrier to treat neurodegenerative diseases. Journal of Controlled Release 235, 34-47.

[39] Hörmann K, Zimmer A (2016) Drug delivery and drug targeting with parenteral lipid nanoemulsions - a review. Journal of Controlled Release 223, 85-98.

[40] Lee MH, Shin GH, Park HJ (2018) Solid lipid nanoparticles loaded thermoresponsive pluronic-xanthan gum hydrogel as a transdermal delivery system. Journal of Applied Polymer Science 135, 46004.

[41] Desfrançois C, Auzély R, Texier I (2018) Lipid Nanoparticles and Their Hydrogel Composites for Drug Delivery: A Review. Pharmaceuticals 11, 118.

[42] Shaikh J, Ankola DD, Beniwal V, Singh D, Kumar MR (2009) Nanoparticle encapsulation improves oral bioavailability of curcumin by at least 9-fold when compared to curcumin administered with piperine as absorption enhancer. European Journal of Pharmaceutical Sciences 37, 223-230.

[43] Zhao L, Du J, Duan Y, Zhang H, Yang C, Cao F, Zhai G (2012) Curcumin loaded mixed micelles composed of Pluronic $\mathrm{P} 123$ and F68: preparation, optimization and in vitro characterization. Colloids and Surfaces B: Biointerfaces 97, 101-108.

[44] Kumar SSD, Mahesh A, Mahadevan S, Mandal AB (2014) Synthesis and characterization of curcumin loaded polymer/lipid based nanoparticles and evaluation of their antitumor effects on MCF-7 cells. Biochimica et Biophysica Acta (BBA)-General Subjects 1840, 1913-1922.

[45] Hong W, Shi H, Qiao M, Zhang Z, Yang W, Dong L, Kang L (2017) pH-sensitive micelles for the intracellular co-delivery of curcumin and Pluronic L61 unimers for synergistic reversal effect of multidrug resistance. Scientific Reports 7, 42465.

[46] Lyrawati D, Trounson A, Cram D (2011) Expression of GFP in the mitochondrial compartment using DQAsome-mediated delivery of an artificial mini-mitochondrial genome. Pharm Res 28, 2848-2862.

[47] Cruz LJ, Stammes MA, Que I, van Beek ER, Knol-Blankevoort VT, Snoeks TJ, Löwik CW (2016) Effect of PLGA NP size on efficiency to target traumatic brain injury. Journal of Controlled Release 223, 31-41.

[48] Choi CHJ, Alabi CA, Webster P, Davis ME (2010) Mechanism of active targeting in solid tumors with transferrin-containing gold nanoparticles. Proceedings of the National Academy of Sciences 107, 1235-1240. 
[49] Wiley DT, Webster P, Gale A, Davis ME (2013) Transcytosis and brain uptake of transferrin-containing nanoparticles by tuning avidity to transferrin receptor. Proceedings of the National Academy of Sciences 110, 8662-8667.

[50] Huang X, Li L, Liu T, Hao N, Liu H, Chen D, Tang F (2011) The shape effect of mesoporous silica nanoparticles on biodistribution, clearance, and biocompatibility in vivo. ACS nano 5, 5390-5399.

[51] Bramini M, Ye D, Hallerbach A, Nic Raghnaill M, Salvati A, Aberg C, Dawson KA (2014) Imaging approach to mechanistic study of nanoparticle interactions with the blood-brain barrier. ACS Nano 8, 4304-4312.

[52] Lockman PR, Koziara JM, Mumper RJ, Allen DD (2004) Nanoparticle surface charges alter blood-brain barrier integrity and permeability. Journal of drug targeting 12,635 641.

[53] Arvizo RR, Miranda OR, Moyano DF, Walden CA, Giri K, Bhattacharya R, Mukherjee $P$ (2011) Modulating pharmacokinetics, tumor uptake and biodistribution by engineered nanoparticles. PloS one 6, e24374.

[54] Anselmo AC, Mitragotri S (2016) Nanoparticles in the clinic. Bioengineering \& translational medicine 1, 10-29. 
Table 1. Hydrodynamic Diameter (d), Polydispersity Index (PDI), Surface Charge, Drug Loading (DL) and Encapsulation Efficiency (EE) of drug-loaded P68 and P68 + DQA nanoformulations prepared at $80^{\circ} \mathrm{C}$ (mean \pm S.D. $n=6$ )

\begin{tabular}{|c|c|c|c|c|c|c|c|}
\hline Sample & Conte & & $d(\mathrm{~nm})$ & PDI & Charge (mV) & $\mathrm{DL}(\%)$ & EE (\%) \\
\hline P68 - CU & $\begin{array}{l}\text { P68 } \\
\text { CU } \\
\text { DFO }\end{array}$ & $\begin{array}{l}10 \mathrm{mg} / \mathrm{ml} \\
2 \mathrm{mg} / \mathrm{ml} \\
-\end{array}$ & $184.3 \pm 12.6$ & $0.124 \pm 0.040$ & $-5.88 \pm 5.74$ & $13.49 \pm 3.17$ & $78.57 \pm 21.26$ \\
\hline $\begin{array}{l}\text { P68 - CU+ } \\
\text { DFO }\end{array}$ & $\begin{array}{l}\text { P68 } \\
\text { CU } \\
\text { DFO }\end{array}$ & $\begin{array}{l}10 \mathrm{mg} / \mathrm{ml} \\
0.28 \mathrm{mg} / \mathrm{ml} \\
5 \mathrm{mg} / \mathrm{ml}\end{array}$ & $177.2 \pm 14.9$ & $0.068 \pm 0.029$ & $-0.44 \pm 2.72$ & $\begin{array}{l}\text { CU: } 1.40 \pm 0.25 \\
\text { DFO: } 24.26 \pm \\
3.18\end{array}$ & $\begin{array}{l}\text { CU: } 66.91 \pm \\
10.40 \\
\text { DFO: } 65.63 \pm \\
11.10 \\
\end{array}$ \\
\hline P68 - DFO & $\begin{array}{l}\text { P68 } \\
\text { CU } \\
\text { DFO }\end{array}$ & $\begin{array}{l}10 \mathrm{mg} / \mathrm{ml} \\
- \\
2 \mathrm{mg} / \mathrm{ml} \\
\end{array}$ & $73.1 \pm 12.5$ & $0.217 \pm 0.021$ & $-2.22 \pm 2.08$ & $15.98 \pm 0.92$ & $95.17 \pm 6.54$ \\
\hline $\begin{array}{l}\text { P68+DQA - } \\
C U\end{array}$ & $\begin{array}{l}\text { P68 } \\
\text { DQA } \\
\text { CU } \\
\text { DFO } \\
\end{array}$ & $\begin{array}{l}9 \mathrm{mg} / \mathrm{ml} \\
1 \mathrm{mg} / \mathrm{ml} \\
2 \mathrm{mg} / \mathrm{ml} \\
-\end{array}$ & $182.6 \pm 31.5$ & $0.099 \pm 0.085$ & $4.27 \pm 4.15$ & $14.68 \pm 1.55$ & $86.17 \pm 10.58$ \\
\hline $\begin{array}{l}\text { P68+DQA - } \\
C U \text { + DFO }\end{array}$ & $\begin{array}{l}\text { P68 } \\
\text { DQA } \\
\text { CU } \\
\text { DFO }\end{array}$ & $\begin{array}{l}9 \mathrm{mg} / \mathrm{ml} \\
1 \mathrm{mg} / \mathrm{ml} \\
0.28 \mathrm{mg} / \mathrm{ml} \\
5 \mathrm{mg} / \mathrm{ml}\end{array}$ & $191.8 \pm 45.3$ & $0.078 \pm 0.036$ & $9.29 \pm 5.12$ & $\begin{array}{l}\text { CU: } 1.01 \pm \\
0.19 \\
\text { DFO: } 31.77 \pm \\
1.80\end{array}$ & $\begin{array}{l}\text { CU: } 81.78 \pm \\
10.16 \\
\text { DFO: } 95.56 \pm \\
7.83\end{array}$ \\
\hline $\begin{array}{l}\text { P68+DQA - } \\
\text { DFO }\end{array}$ & $\begin{array}{l}\text { P68 } \\
\text { DQA } \\
\text { CU } \\
\text { DFO }\end{array}$ & $\begin{array}{l}9 \mathrm{mg} / \mathrm{ml} \\
1 \mathrm{mg} / \mathrm{ml} \\
- \\
2 \mathrm{mg} / \mathrm{ml}\end{array}$ & $50.44 \pm 33.1$ & $0.246 \pm 0.048$ & $0.02 \pm 1.62$ & $16.10 \pm 0.43$ & $95.94 \pm 3.07$ \\
\hline
\end{tabular}




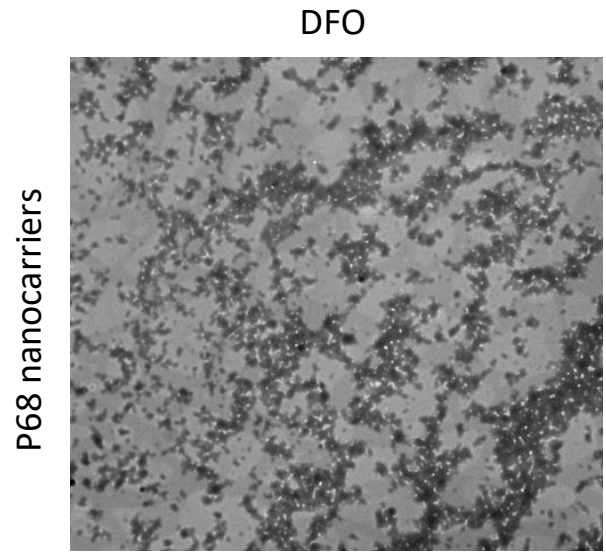

$-500 \mathrm{~nm}$

$H V=120.0$ kV, Direct Mag: 17500x

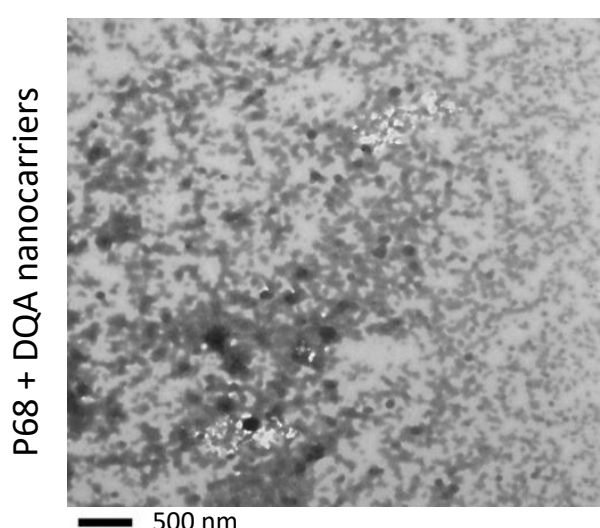

$H V=120.0$ kV, Direct Mag: 17500x

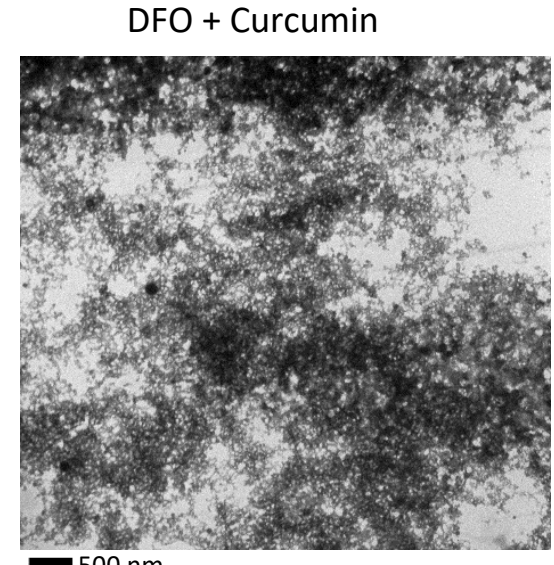

$-500 \mathrm{~nm}$

$H V=120.0$ kV, Direct Mag: 17500x

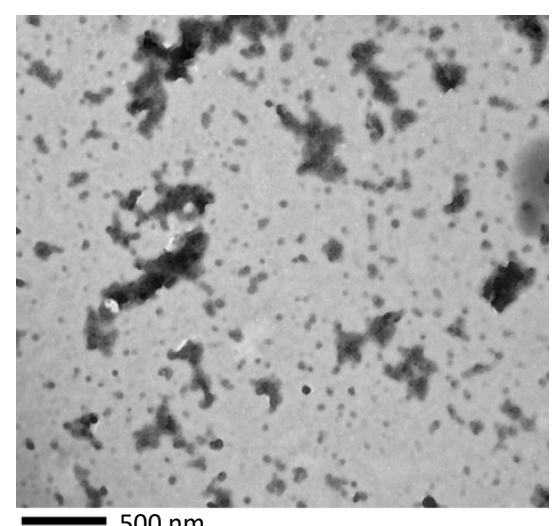

$\mathrm{HV}=120.0 \mathrm{kV}$, Direct Mag: 33500x
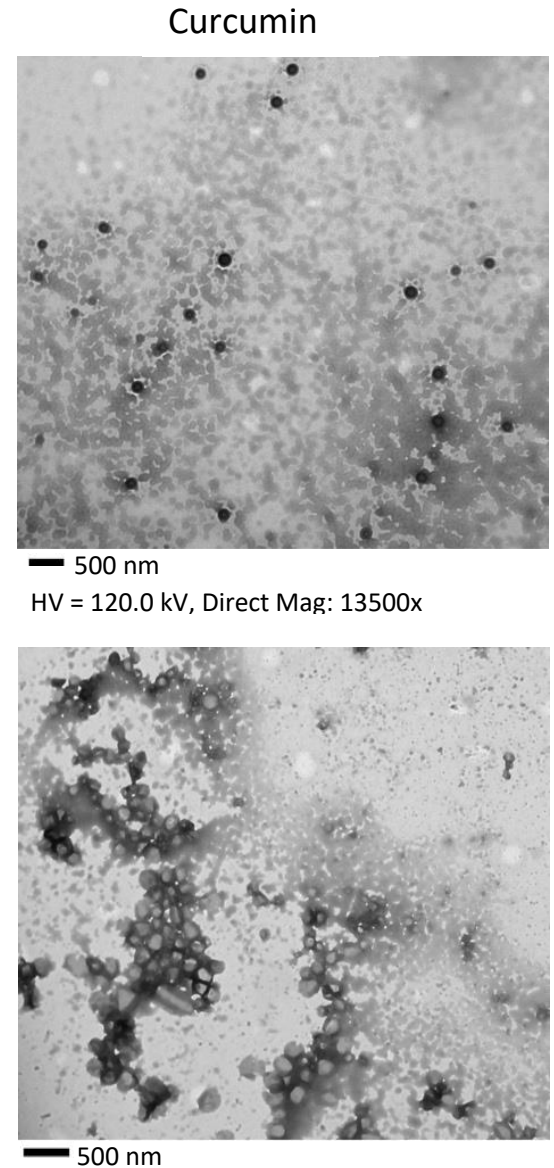

HV $=120.0 \mathrm{kV}$, Direct Mag: 17500x

Figure 1. TEM images of P68 and P68 DQA drug-loaded nanocarriers. Scale $=500 \mathrm{~nm}$ in all cases. 

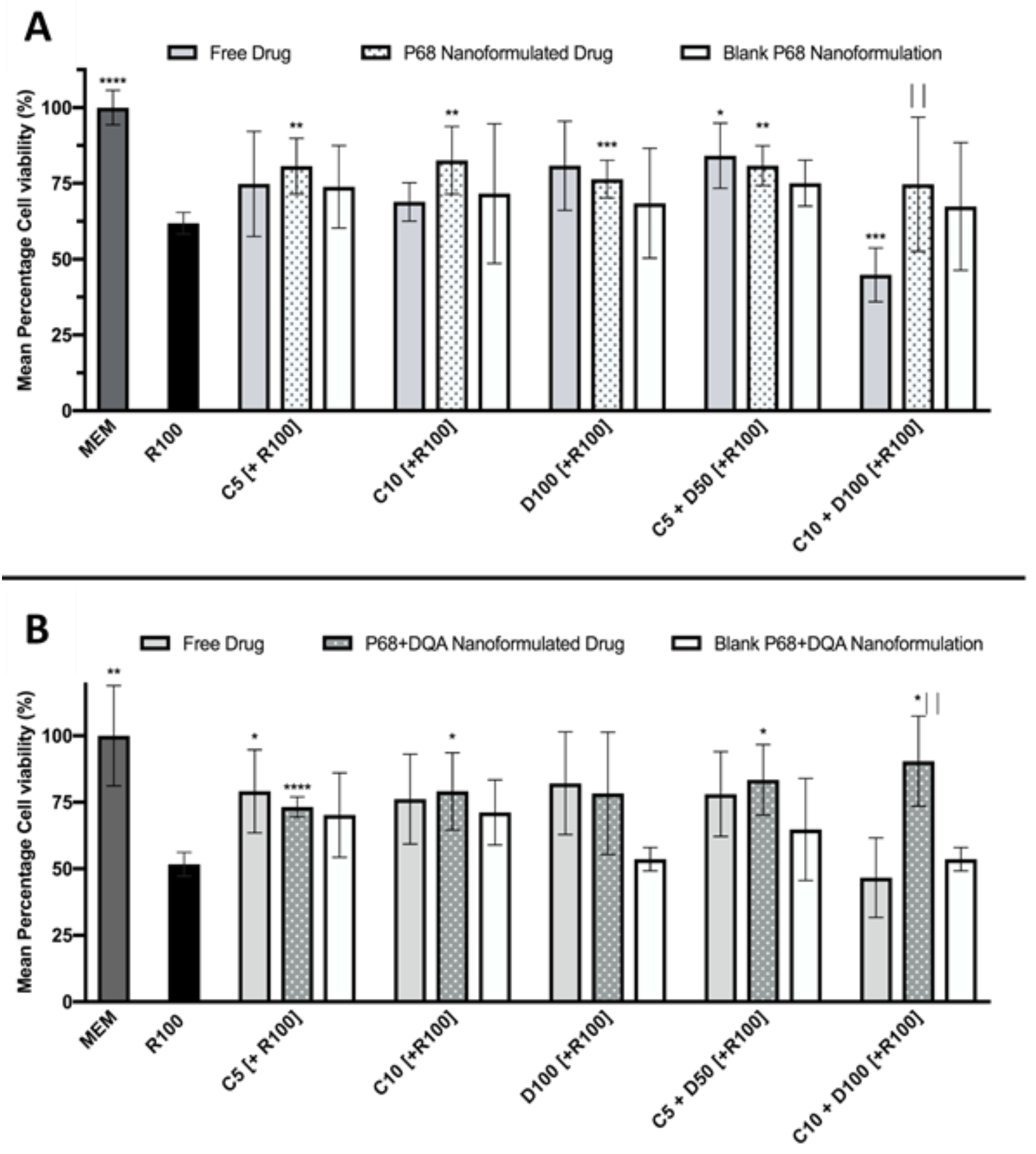

Figure 2. A. MTT assay results of 3-hour pre-treatment with free drug, P68 nanoformulated or corresponding blank preparations of either curcumin $(C 5, C 10)$, deferoxamine (D100) or combined curcumin and deferoxamine $(C 5+D 50$, $C 10+D 100)$ followed by 24-hour treatment with 100 uM rotenone (R100) compared to R100 treatment alone. MEM represents the control condition where cells were only treated with media, no pre-treatment nor $R 100$ treatment $(n=6)$. $B$. Corresponding MTT assay results for P68+DQA nanoformulated pre-treatments $(n=6) . *$ represents significance values of control or pre-treatment conditions compared to R100 treatment alone $(* * * *=p<0.0001, * * *=p<0.001, * *=p<0.01, *$ $=p<0.05)$. | represents significance values of nanoformulated drug compared to free drug within the same treatment condition ( $\mid=p<0.01)$. 

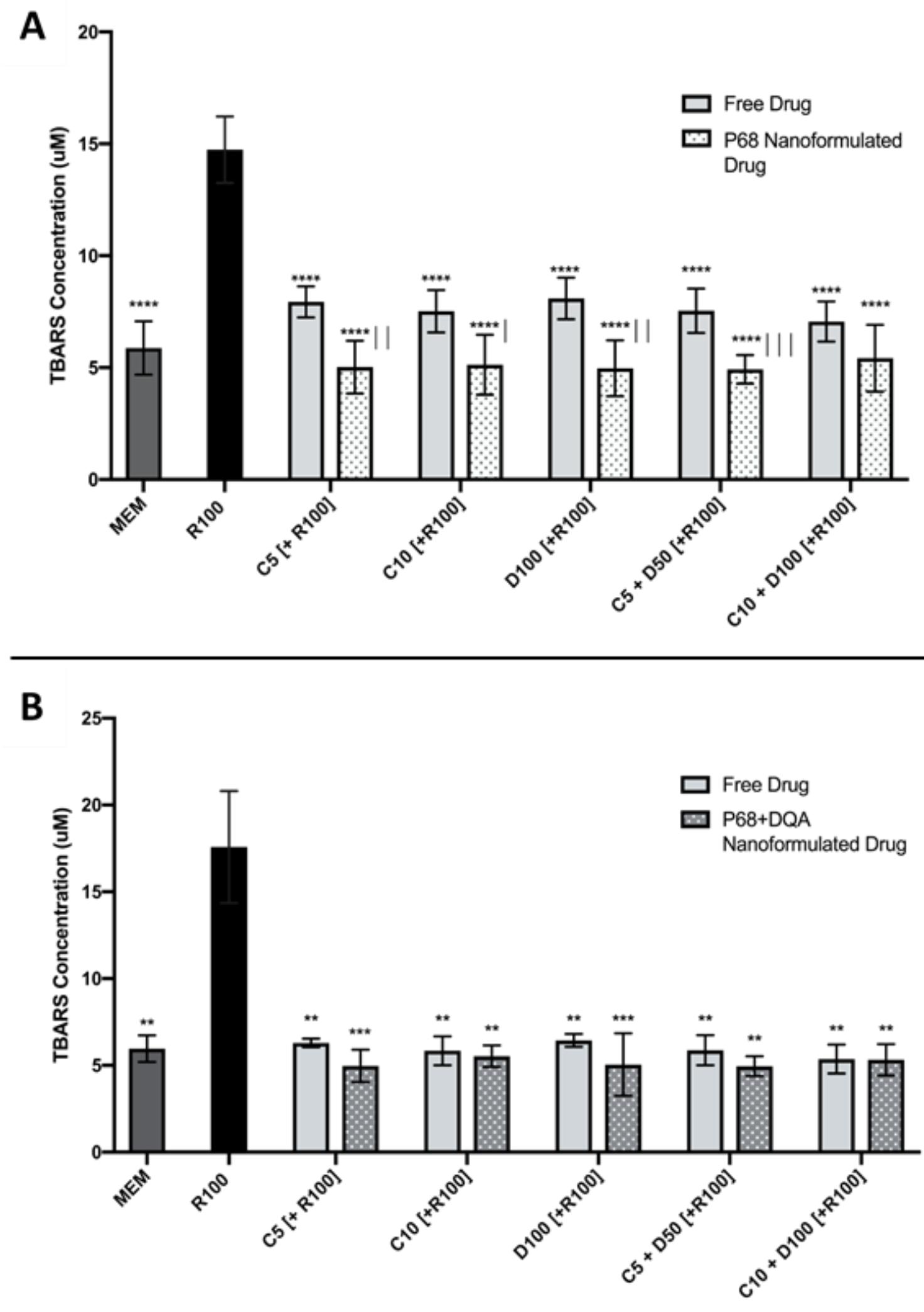

Figure 3. A. TBARS assays results of 3-hour pre-treatment with free drug or P68 nanoformulated preparations of either curcumin $(C 5, C 10)$, deferoxamine $(D 100)$ or combined curcumin and deferoxamine $(C 5+D 50, C 10+D 100)$ followed by 24 hour treatment with 100 uM rotenone (R100) compared to R100 treatment alone. MEM represents the control condition where cells were only treated with media, no pre-treatment nor $R 100$ treatment $(n=6)$. B. Corresponding TBARS assay results for $P 68+D Q A$ nanoformulated pre-treatments $(n=6) . *$ represents significance values of control or pre-treatment conditions compared to $R 100$ treatment alone $(* * * *=p<0.0001, * * *=p<0.001, * *=p<0.01)$. | represents significance values of nanoformulated drug compared to free drug within the same treatment condition $(|||=p<0.001, \|=p<0.01|$, $=p<0.05)$. 


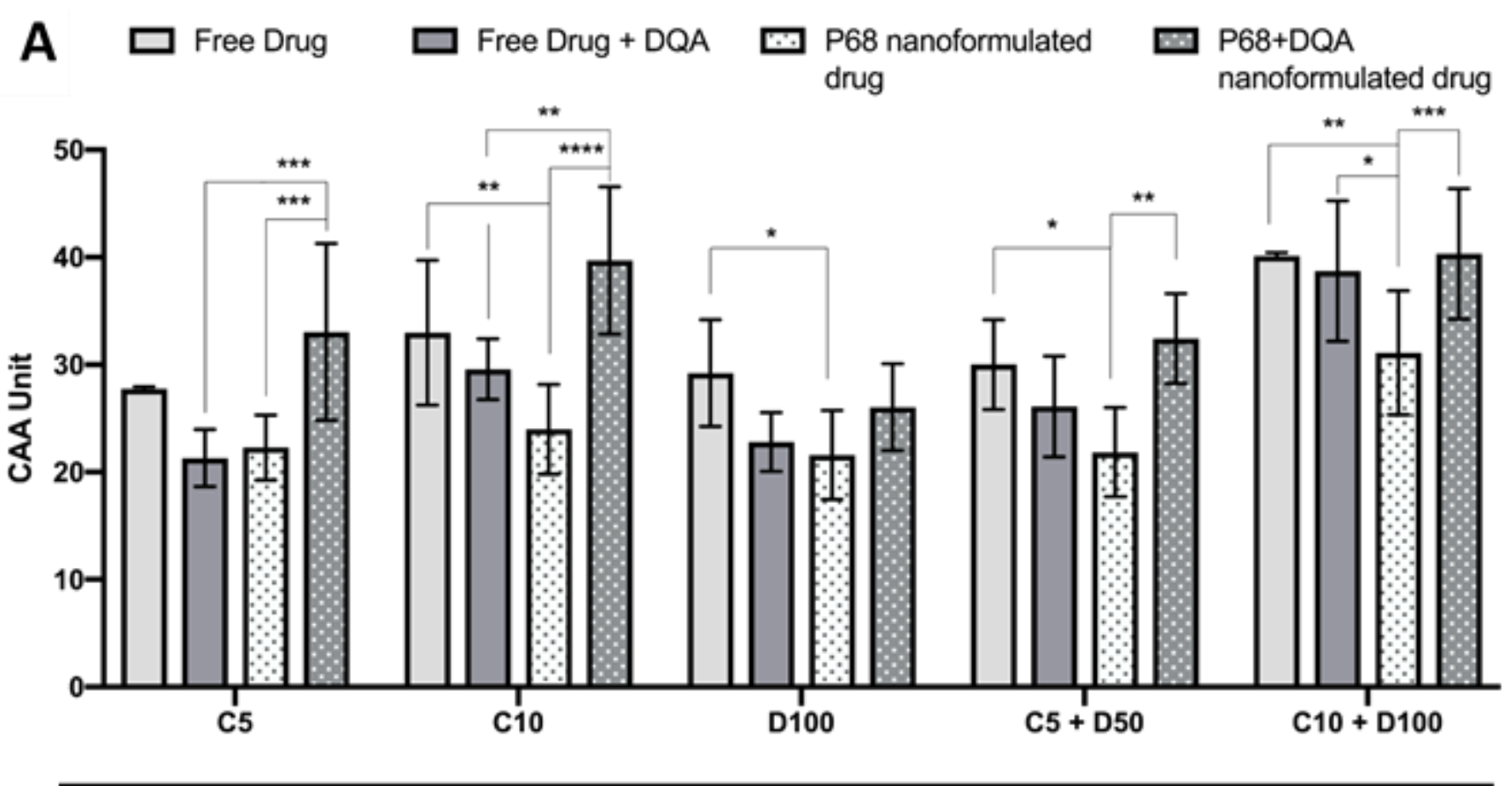

B

\begin{tabular}{llcc}
\hline Preparation & Condition comparison & $\begin{array}{c}\text { Mean difference } \\
\text { (CAA unit) }\end{array}$ & $\begin{array}{c}\text { Significance } \\
(p \text {-value })\end{array}$ \\
\hline Free Drug & C10+D100 vs. C5 & 12.35 & 0.0002 \\
& C10+D100 vs. D100 & 10.89 & 0.0013 \\
& C10+D100 vs. C5+D50 & 10.1 & 0.0036 \\
\hline Free Drug + DQA & C10 vs. C5 & 8.27 & 0.0274 \\
& C10+D100 vs. C5 & 17.42 & $<0.0001$ \\
& C10+D100 vs. C10 & 9.15 & 0.0108 \\
& C10+D100 vs. D100 & 15.92 & $<0.0001$ \\
& C10+D100 vs. C5+D50 & 12.61 & 0.0001 \\
\hline P68 nanoformulated & C10+D100 vs. C5 & 8.81 & 0.0156 \\
drug & C10+D100 vs. D100 & 9.51 & 0.0072 \\
& C10+D100 vs. C5+D50 & 9.25 & 0.0096 \\
\hline P68 + DQA & C10 vs. D100 & 13.65 & $<0.0001$ \\
nanoformulated drug & C10+D100 vs. D100 & 14.28 & $<0.0001$ \\
& C10+D100 vs. C5+D50 & 7.89 & 0.0400 \\
\hline
\end{tabular}

Figure 4. A. CAA assay results for free drug, free drug + DQA, P68 \& P68 DQA preparations of curcumin (C5, C10), deferoxamine (D100) and combined curcumin and deferoxamine $(C 5+D 50, C 10+D 100) .{ }^{*}$ represent significance values within drug conditions $\left.{ }^{* * * *}=p<0.0001, * * *=p<0.001, * *=p<0.01, *=p<0.05\right)$. B. A table to show the corresponding significant CAA results between drug conditions of the same treatment preparation. 


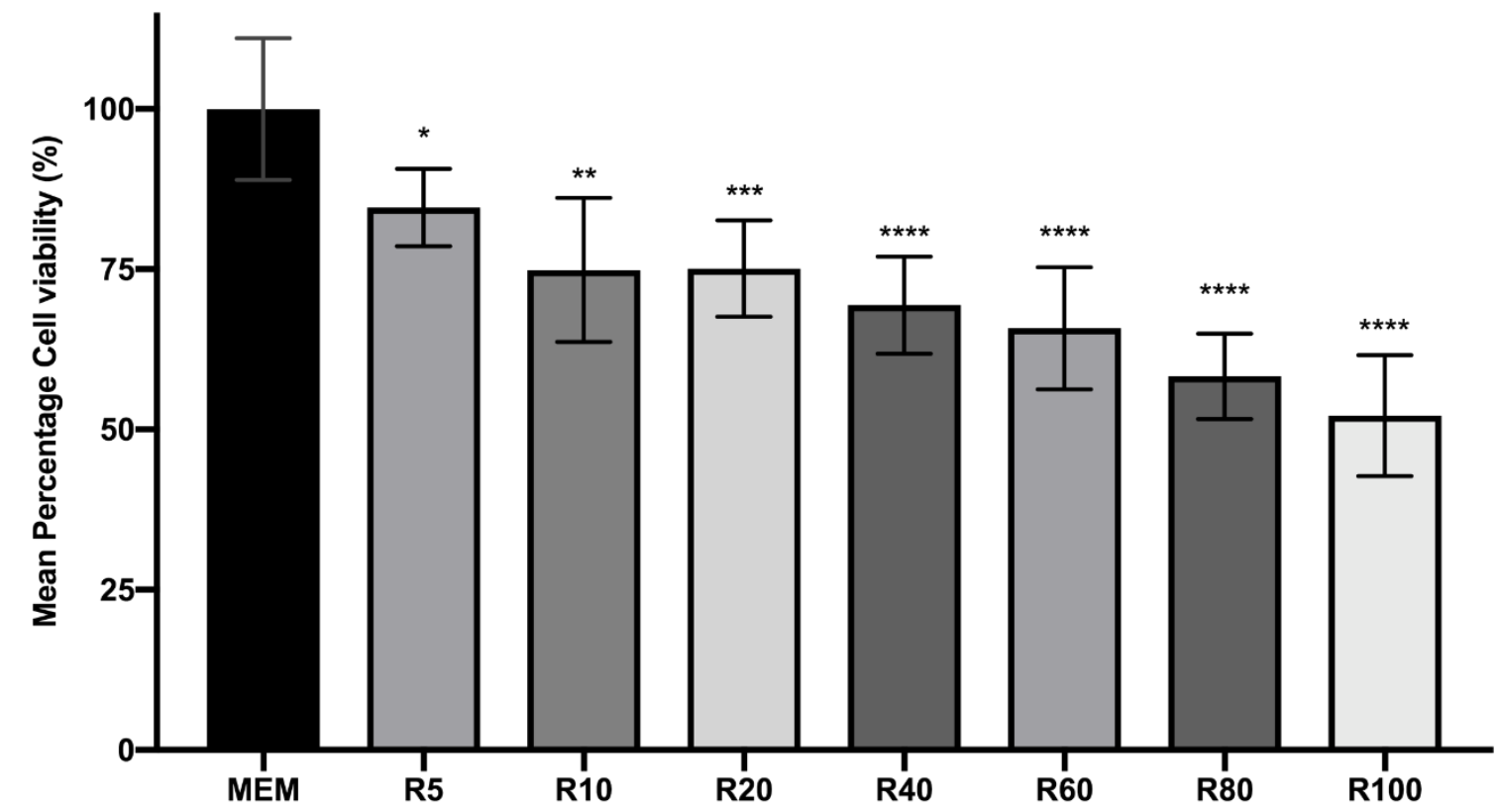

Supplementary figure 1. MTT assay results of 24-hour 5uM-100uM rotenone treatment. MEM represents the control condition where cells were only treated with media $(n=6) . *$ represents significance values of the treatment conditions compared to the control condition $(* * * *=p<0.0001, * * *=p<0.001, * *=p<0.01, *=p<0.05$ ). 